\title{
Relações entre controle executivo e memória episódica verbal no comprometimento cognitivo leve e na demência tipo Alzheimer
}

\author{
Amer Cavalheiro Hamdan \\ Universidade Federal do Paraná \\ Orlando Francisco Amodeo Bueno \\ Universidade Federal de São Paulo
}

\begin{abstract}
Resumo
Este estudo comparou e relacionou o desempenho de idosos com Comprometimento Cognitivo Leve e Demência Tipo Alzheimer em testes de controle executivo e de memória episódica verbal. Para a avaliação da memória episódica verbal utilizamos a Tarefa de Recordação de Palavras Imediata e a Tarefa de Recordação de Palavras com Intervalo. Na avaliação do controle executivo foram utilizados, os seguintes testes: Random Number Generation, Trail Making Test, Fluência Verbal semântica e fonológica, Wisconsin Card Sorting Test, Reading Span Test e Brow-Peterson Test. Treze idosos do grupo controle foram equiparados quanto à idade e escolaridade com nove idosos com Comprometimento Cognitivo Leve e oito idosos com Demência Tipo Alzheimer. Foram encontradas diferenças e associações estatisticamente significantes em relação aos testes de controle executivo entre os grupos investigados. Apesar da presença de associações significativas entre a memória episódica verbal e o controle executivo, não foram evidenciados déficits do controle executivo nos idosos com Comprometimento Cognitivo Leve.
\end{abstract}

Palavras-chave: neuropsicologia; alzheimer; controle executivo; memória episódica

\begin{abstract}
Relationships between executive control and verbal episodic memory in the mild cognitive impairment and the Alzheimer-type dementia. This study compared and related the performance of older people with Mild Cognitive Impairment and Alzheimer-type dementia in tests of executive control and of verbal episodic memory. In the evaluation of the verbal episodic memory we used the Task of Immediate Word Recall and the Task of Delayed Word Recall. In the evaluation of executive control we used the following tests: Random Number Generation, Trail Making Test, Semantic and Phonological Verbal Fluency, Wisconsin Card Sorting Test, Reading Span Test, and Brow-Peterson Test. Thirteen elders of the control group were compared by age and education with nine elders with Mild Cognitive Impairment and eight elders with Alzheimer-type dementia. Differences and significant statistical associations were found in relation to the tests of executive control with the groups investigated. Despite the presence of significant associations between the verbal episodic memory and the executive control, deficits of the executive control were not evidenced in groups with Mild Cognitive Impairment.
\end{abstract}

Keywords: neuropsychology; alzheimer; executive control; episodic memory

$\mathrm{F}$ unções executivas (FE) referem-se às habilidades cognitivas envolvidas no planejamento, iniciação, seguimento e monitoramento de comportamentos complexos dirigidos a um fim. Na avaliação neuropsicológica, o termo FE é utilizado para designar uma ampla variedade de funções cognitivas que implicam: atenção, concentração, seletividade de estímulos, capacidade de abstração, planejamento, flexibilidade, controle mental, autocontrole e memória operacional. Inúmeros testes e baterias neuropsicológicas têm sido empregados para avaliar as FEs (Green, 2000; Spreen \& Strauss, 1998).
Evidências advindas da avaliação neuropsicológica (Stuss \& Alexander, 2000; Stuss \& Levine, 2002), de neuroimagem (Carpenter, Just, \& Reichle, 2000; Royall et al., 2002; Smith \& Jonides, 1999) e de pesquisas neurofisiológicas (Funahashi, 2001) têm apontado para a necessidade atual de fracionamento das FEs, diferenciando o produto do mecanismo de execução. Em outras palavras, FEs podem ser compreendidas como um termo amplo, que se refere ao produto de uma operação eliciada por vários processos cognitivos para realizar uma tarefa particular. Por sua vez, o controle executivo (CE) pode ser compreendido como um sistema ou meca- 
nismo responsável pela coordenação dos vários processos implicados na realização das FEs. O lobo frontal, em particular a região pré-frontal, tem sido relacionado com o processamento cognitivo das FEs e do CE. Lesões nessas regiões e nos circuitos corticais e subcorticais ocasionam diferentes síndromes neuropsicológicas (Faw, 2003; Tekin \& Cummings, 2002). Por esta razão, há necessidade de cuidados na avaliação neuropsicológica do CE, pois, as relações entre estrutura e função, sistemas e processos, lesão e comprometimento funcional e entre fisiologia e função cognitiva, ainda não estão plenamente estabelecidas (Royall et al., 2002; Stuss \& Levine, 2002; Tirapu-Ustárroz, Munõz-Céspedes, Pelegrin-Valero, 2002).

No envelhecimento normal tem sido constatado alteração ou déficit em relação à memória episódica verbal e ao CE, e não há uma compreensão clara deste processo. No envelhecimento, os déficits nas tarefas neuropsicológicas da memória episódica e do CE ocorrem, principalmente, associados à diminuição no processamento da informação, nos processos atencionais, nos processos inibitórios e na flexibilidade cognitiva (Grady \& Craik, 2000; Green, 2000; Woodruff-Pak, 1997). Explicação para essas alterações cognitivas associadas ao envelhecimento tem sido formulada em termos de uma hipótese do envelhecimento do sistema frontal (Stebbins et al., 2002; West, 1996). Segundo esse modelo, no envelhecimento normal ocorre um declínio das funções cognitivas relacionadas às habilidades verbais, visuais e temporais, em decorrência do processo de envelhecimento fisiológico natural dos lobos frontais. Esse modelo tem encontrado sustentação nas pesquisas neuropsicológicas e neurobiológicas sobre os efeitos do envelhecimento nos processos cognitivos (Grady \& Craik, 2000).

A Demência Tipo Alzheimer (DTA) é uma doença crônico-degenerativa que atinge o Sistema Nervoso Central, manifestando-se, inicialmente, através da perda de memória para fatos recentes, e, posteriormente, atingindo outras funções cognitivas como a linguagem e as funções executivas. A DTA compromete as atividades sociais e funcionais do indivíduo (Associação Psiquiátrica Americana, 1995) e atinge, igualmente, homens e mulheres (Barnes et al., 2003). O Comprometimento Cognitivo Leve (CCL) é um estado intermediário entre o envelhecimento normal e a DTA, cuja característica principal é o comprometimento da memória episódica, sem outros déficits cognitivos e funcionais, e que apresentam um risco aumentado de desenvolver demência (Petersen et al., 1999; 2001). Em outras palavras, o CCL é uma condição clínica da pessoa que apresenta perda de memória episódica maior que a esperada para a sua idade e na qual esta condição não satisfaz critérios para a Doença de Alzheimer provável (para revisão do conceito de CCL, ver, e.g., Bischkop, Busse, \& Angermeyer, 2002; Petersen et al., 2001). As pesquisas em idosos com CCL são importantes na medida em que aumenta a população de idosos, crescendo também as queixas e os problemas de memória em ambulatórios e serviços de saúde. Esses idosos poderão ser beneficiados por tratamentos, em estágio anterior à manifestação plena da demência. A Associ- ação Americana de Psicologia e a Academia Americana de Neurologia recomendam que pessoas com CCL devam ser identificadas e monitoradas quanto à progressão para desenvolver DTA (American Psychological Association, 1998; Petersen et al., 2001).

Vários estudos têm procurado identificar e predizer quais indivíduos irão desenvolver demência no futuro, não apenas com marcadores fisiopatológicos (Buerger et al., 2002; Riemenschneider et al., 2002), mas também tendo como base parâmetros neuropsicológicos (Collie \& Maruff, 2000; Palmer, Bäckman, Winblad, \& Fratiglioni, 2003). Em recente estudo (Petersen et al., 2001), que utilizou a metodologia da revisão baseada em evidências, foi recomendada tanto a utilização de instrumentos de rastreios (e.g., Mini-Exame do Estado Mental - MEEM e Teste do Desenho do Relógio), como a utilização de baterias neuropsicológicas, instrumentos cognitivos breves e entrevistas do informante estruturadas para a identificação e monitoramento de pessoas com CCL.

Na DTA, comprometimento do CE tem sido evidenciado de maneira inequívoca, principalmente nos estágios iniciais e com a progressão da doença, mas este processo não está plenamente compreendido (para uma revisão, ver, e.g., Perry \& Hodges, 1999). As evidências do comprometimento do CE no CCL também têm sido observadas, mas ainda existem poucos trabalhos a respeito (e.g., Hänninen et al., 1997). A fluência verbal semântica, a denominação de objetos, a solução de problemas, as tarefas visuoconstrutivas e a atenção encontram-se comprometidas na DTA (Foster, Behrmann, \& Stuss, 1999; Green, 2000). Vários estudos têm indicado o comprometimento da memória operacional na DTA em diferentes tipos de material e procedimentos (para uma revisão, ver, e.g., Morris, 1994). Outra linha de investigação do CE na DTA tem estudado o processamento de sentenças, mas ainda as evidências são pequenas. Os processos cognitivos no processamento de sentenças envolvem medidas de controle inibitório, planejamento e memória operacional. Algumas evidências têm demonstrado que a compreensão de sentenças na DTA está comprometida (Grossman \& Rhee, 2001; Grossman \& WhiteDevine, 1998). Outros trabalhos têm procurado demonstrar as diferenças de comprometimento do CE na DTA em relação a outros domínios cognitivos e patologias (Binetti et al., 1996; Yuspeh, Vanderploeg, Crowell, \& Mullan, 2002).

As evidências apresentadas anteriormente sugerem que no envelhecimento, e em particular, na DTA ocorre o declínio da memória episódica verbal e do CE, mas não há estudos mostrando as relações entre o declínio da memória episódica verbal e o CE. Com este estudo talvez se possa compreender melhor a relação entre o comprometimento da memória episódica verbal e outros sistemas cognitivos e também a fronteira entre o envelhecimento normal e o patológico. Este estudo teve dois objetivos. O primeiro foi comparar o desempenho de idosos com CCL e com DTA em alguns testes de controle executivo. O segundo objetivo foi relacionar o comprometimento da memória episódica verbal com o desempenho nos testes de controle executivo nestes grupos de idosos. 


\section{Método}

\section{Participantes}

Participaram desse estudo trinta idosos, de ambos os sexos, acima de 60 anos divididos em três grupos: (a) indivíduos com CCL ( $n=09$ ); (b) indivíduos com DTA ( $n=08)$; (c) grupo controle $(n=13)$. A amostra foi selecionada a partir de uma triagem de 123 idosos avaliados no Ambulatório de Saúde Mental, do Hospital Universitário da Universidade Federal de Mato Grosso do Sul/ UFMS. Para o grupo controle foram incluídos sujeitos que procuraram o ambulatório com queixas de problemas de memória que, do ponto de vista clínico, podiam ser considerados saudáveis. Os critérios de exclusão foram: (1) idade abaixo de 60 anos; (2) presença de déficit visual ou auditivo não-corrigido; (3) histórico de alcoolismo ou exposição a toxinas; (4) uso crônico de medicamentos psicoativos (benzodiazepínicos, neurolépticos, etc.); (5) presença de anormalidades endócrinas e metabólicas; (6) alterações neurológicas (parkinsonismo, traumatismo crâneoencefálico, doença cerebrovascular, etc.); (7) presença de distúrbios psiquiátricos (distúrbios de ansiedade, distúrbios de pensamento, etc.) e (8) indivíduos analfabetos. Para o diagnóstico de DTA empregamos os critérios estabelecidos pelo DSM-IV (Associação Psiquiátrica Americana, 1995). Os critérios clínicos utilizados para CCL foram: (a) queixa de problemas de memória, preferencialmente corroborada por um informante; (b) declínio da memória comparada à idade e educação; (c) função cognitiva geral preservada; (d) atividades da vida diária intactas; (e) não preenchimento dos critérios de demência (Petersen et al., 1999).

A idade média da amostra de 30 idosos foi 69,5 ( $\pm 5,3$ anos) e o intervalo de confiança (IC, 95\%) de 67,4-71,5 anos. A idade mínima foi 61 anos e a idade máxima 84 anos, sendo 18 idosos do sexo feminino (60\%) e 12 do sexo masculino (40\%). Quanto à escolaridade, a média foi de 3,08 ( $\pm 2,2$ anos) de estudo e o intervalo de confiança IC (95\%) de 2,9-4,6 anos. A escolaridade mínima foi de 01 ano e a máxima de 08 anos. A Tabela 1 apresenta a distribuição dos grupos controle, CCL e DTA em relação à idade e escolaridade. A ANOVA não revelou diferenças significantes entre os grupos em relação à idade e a anos de escolaridade.

\section{Material}

Instrumentos para triagem e avaliação da memória episódica verbal: Entrevista Neuropsicológica Estruturada: instrumento elaborado para levantar informações gerais sobre dados pessoais, estado geral de saúde, hábitos de leitura, escrita, atividades físicas e sociais (American Psychological Association, 1998; Green, 2000). Exame do Estado Mental: procedimento para avaliação do estado mental (Trzepacs \& Baker; 1993). Mini-Exame do Estado Mental (MEEM): empregado para rastrear a presença ou não de comprometimento cognitivo (Folstein, Folstein \& Mchugh, 1975). Escala de Depressão Geriátrica (GDS): o objetivo deste instrumento é a avaliação do estado afetivo, diferenciando os indivíduos com e sem sintomas de depressão. Utilizamos a forma reduzida em quinze questões, identificando os sintomas de depressão e os fatores de risco (Yesavage et al., 1983). Escala Clínica de Demência (CDR): empregada para discriminar os estágios da demência, em cinco graus: sem demência (CDR $=0$ ), demência questionável (CDR $=0,5)$, demência leve $(C D R=1)$, demência moderada $(C D R=2)$ e demência grave $(C D R=3)$. Os domínios avaliados incluem: memória, orientação, juízo e solução de problemas, assuntos comunitários, casa, hobbies e cuidados pessoais (Morris, 1993). Teste do Desenho do Relógio (TDR): empregado para triagem cognitiva (Spreen \& Strauss, 1998). Para avaliação da memória episódica verbal utilizamos o Teste de Recordação de Palavras Imediatas (Repai) e o Teste de Recordação de Palavras com Intervalo (Repaci), ambos retirados da Bateria ADAS-COG (adaptada por Schultz, Siviero, \& Bertolucci, 2001). O teste consiste em solicitar ao participante para ler em voz alta uma lista de 10 palavras, recordando-as imediatamente. No decorrer dos próximos cinco minutos, aplica-se uma tarefa distrativa (Digit Span), após o que, solicita-se aos participantes que se recordem, novamente, das palavras lidas anteriormente. A pontuação foi dada pelo número total de palavras recordadas em três ensaios realizados (Repai) e pelo número de palavras recordadas após a tarefa distrativa (Repaci). Utilizamos pontos de cortes propostos por Bertolucci et al. (2001) para idosos com DTA. O Repai e Repaci foram utilizados como medidas de aquisição e recuperação da memória episódica verbal. Digit Span (DS): parte integrante da Escala de Memória de Wescheler -revisada (WMS-r) foi utilizada para testar a habilidade do sujeito repetir seqüências de números em ordem direta e depois em ordem inversa (Wechsler, 1981).

Instrumentos para avaliação do Controle Executivo: Random Number Generation (RNG): consiste em solicitar ao participante que fale em voz alta, aleatoriamente, números de 01 a 10. A pontuação é dada pelo índice de Evans (Hamdan, Bueno, \& Souza, 2004). Trail Making Test (TMT): na forma A apresentam-se ao sujeito círculos com números de 1 a 25, impressos numa folha, que devem ser ligados em ordem crescente. Na forma B estão impressos números e letras que o sujeito deve ligar alternadamente. A pontuação é dada pelo tempo gasto para completar a tarefa (Spreen \& Strauss, 1998). Fluência Verbal - Semântica: foi aplicado utilizando a categoria frutas. $\mathrm{O}$ teste consiste em solicitar ao sujeito que fale o maior número de nomes de frutas de que se recorde, durante 1 minuto. A pontuação é dada pelo número de palavras citadas (Spreen \& Strauss, 1998). Fluência Verbal - Fonológica: consiste em solicitar ao sujeito para recordar todas as palavras iniciadas com as letras /F/; /A/ e /S/ durante um minuto. A pontuação é dada pela soma de todas as palavras recordadas nas três letras (Spreen \& Strauss, 1998). Wisconsin Card Sorting Test (WCST): o teste é composto por cartas com figuras geométricas que variam de cor e número, retiradas de um conjunto. Essas devem ser associadas a quatro cartõesestímulo, por um critério a ser descoberto pelo sujeito. A pontuação é dada pelo número total de categorias completadas e pelo número total de erros cometidos (Spreen \& Strauss, 1998). Reading Span (Rspan): empregamos uma adaptação do teste 
desenvolvido para medir o span de working memory durante a leitura (Daneman \& Carpenter, 1980). O teste consiste em ler uma seqüência de frases (num total de 76) que variam em extensão, estruturadas em 04 níveis de 05 séries. Metade dessas frases contém afirmações verdadeiras e metade afirmações falsas sobre conhecimentos gerais. O participante deverá identificar em cada uma, o critério de verdadeiro ou falso, devendo, ao final, recordar a última palavra de cada sentença. A tarefa implica simultaneamente em processamento e armazenamento. O sujeito tem de ler e compreender as frases e ainda reter as últimas palavras de cada frase. A pontuação é dada pelo número de palavras recordadas da série (Daneman \& Carpenter, 1980). Teste de BrownPeterson: utilizamos uma adaptação do teste desenvolvido originalmente por Brown-Peterson (Brown, 1958; Peterson \& Peterson, 1959). No teste é solicitado aos sujeitos que recordem cinco séries de três letras a intervalos regulares de tempo (1, 3, 9 e 18 segundos) marcados através de leituras de números de ordem inversa. A pontuação é dada pelo número total de séries corretamente recordadas em cada intervalo de tempo (Spreen \& Strauss, 1998).

\section{Procedimento}

Inicialmente, realizamos uma triagem dos sujeitos encaminhados por neurologistas e psiquiatras, ou que procuraram espontaneamente o Ambulatório de Saúde Mental para participar da pesquisa. Esta triagem foi realizada mediante: Entrevista Neuropsicológica Estruturada, Exame do Estado Mental, Escala de Depressão Geriátrica (GDS), Tarefa de Recordação de Palavras, Mini-Exame do Estado Mental (MEEM) e Escala Clínica de Demência (CDR). Com esses procedimentos, muitos pacientes que não preenchiam os critérios previamente estabelecidos foram excluídos. A Entrevista Neuropsicológica Estruturada e o Exame do Estado Mental foram empregados para excluir outras possíveis causas de alterações cognitivas não incluídas nos critérios da pesquisa (foram identificados e excluídos casos de demência vascular, alcoolismo, alterações de humor, etc.).

As tarefas de Recordação de Palavras Imediatas (Repai) e de Recordação de Palavras com Intervalo (Repaci) foram empregadas para evidenciar o comprometimento da memória episódica. Foram classificados os grupos CCL e DTA, de acordo com os pontos de corte estabelecidos por Bertolucci et al. (2001) na tarefa de recordação de uma lista de palavras da bateria CERAD. Deste modo, os indivíduos que obtiveram desempenho $\leq 13$ acertos na soma total do Repai e $\leq 03$ no Repaci foram incluídos nos grupos CCL e DTA. No grupo CCL foram incluídos os sujeitos que apresentaram desempenho abaixo do ponto de corte em pelo menos um dos testes de memória episódica. No grupo controle foram incluídos os sujeitos que obtiveram desempenho acima dos pontos de corte pré-estabelecidos. Para a inclusão nos grupos utilizamos também o ponto de corte no MEEM de 24 pontos ou mais para os grupos controle e CCL e 23 pontos ou menos para o grupo de pacientes com DTA. No GDS, o ponto de corte foi de até 05 pontos para indivíduos sem sintomas depressivos e 06 ou mais para indivíduos com sintomas (ex- cluídos da amostra). Os indivíduos do grupo CCL obtiveram escore de 0,5 no CDR; 1 para o grupo DTA e 0 para o grupo controle. Após a triagem foram aplicados os testes para avaliação do controle executivo.

Para os indivíduos selecionados, sessões foram realizadas, individualmente, com duração em torno de 50 minutos. O número de sessões variou de duas a três, dependendo das características individuais de cada idoso. Todos os participantes foram informados antes do início da investigação sobre os objetivos gerais da pesquisa e assinaram o Termo de Consentimento de Participação, previamente aprovado pelo Comitê de Ética da Universidade Federal de São Paulo (UNIFESP) e pelo Comitê de Pesquisa da Universidade Federal de Mato Grosso do Sul (UFMS).

Para efeito de análise estatística consideramos como variáveis independentes às amostras de idosos com CCL, DTA e controle; e como variáveis dependentes os resultados nos testes de memória episódica verbal e de controle executivo. Os grupos foram emparelhados quanto à idade e escolaridade. Na a análise descritiva dos resultados utilizamos as medidas de tendência central (média) e de dispersão (desvio-padrão). Para a análise inferencial empregamos provas paramétricas (teste de análise de variância - ANOVA e teste de correlação de Pearson) para comparar o desempenho e verificar a associação entre os resultados dos testes de memória episódica verbal e controle executivo. A análise post hoc foi realizada mediante o teste de Tukey para diferenciar os grupos. O valor $p$ foi estabelecido para cada teste e a hipótese nula foi rejeitada ao nível de significância de alfa $=5 \%$.

\section{Resultados}

A Tabela 1 apresenta as médias e os desvios-padrão para os critérios de seleção dos grupos. A ANOVA revelou diferenças estatisticamente significativas em relação ao MEEM, ao TDR, ao Repai e ao Repaci. Porém, não foram observadas diferenças significantes em relação ao GDS e ao DS, tanto para ordem direta quanto inversa. Os resultados da comparação post hoc (teste de Tukey) revelaram que o grupo DTA diferenciou-se significativamente dos grupos controle e CCL no MEEM e no TDR. No Repai, o grupo controle diferenciouse significativamente dos grupos CCL e DTA. Por fim, no Repaci as diferenças ocorreram entre os três grupos.

O estudo de correlação revelou várias associações significativas entre o Repai e Repaci e os critérios de seleção. O Repai foi associado significativamente com a idade $(r=-0,41$; $p=0,023)$, a escolaridade $(r=0,37 ; p=0,041)$, com o MEEM $(r$ $=0,63 ; p=0,000)$, o TDR $(r=0,62 ; p=0,000)$ e o Repaci $(r=$ 0,$83 ; p=0,000$ ). Por sua vez, o Repaci foi associado significativamente com a idade $(r=-0,36 ; p=0,048)$, com o MEEM ( $r$ $=0,81 ; p=0,000)$ e com o TDR $(r=0,72 ; p=0,000)$. Não foi observada correlação significante entre escolaridade e o Repai, mas este apresentou tendência para significância ( $r=$ $0,31 ; p=0,088)$. As magnitudes das correlações encontradas foram de média a forte, entre os testes Repai e Repaci e o MEEM e o TDR, sugerindo a adequação dos critérios utilizados na seleção dos grupos. 
Tabela 1

Características demográficas e desempenho nos testes de triagem e memória episódica verbal dos grupos controle, CCL e DTA

\begin{tabular}{|c|c|c|c|c|c|}
\hline & $\begin{array}{c}\text { CON } \\
(n=13) \\
M \pm D P\end{array}$ & $\begin{array}{c}\text { CCL } \\
(n=9) \\
M \pm D P\end{array}$ & $\begin{array}{c}\text { DTA } \\
(n=8) \\
M \pm D P\end{array}$ & $F$ & $p$ \\
\hline Idade & $67,3 \pm 4,6$ & $70,3 \pm 4,4$ & $72,1 \pm 6,6$ & 2,33 & 0,117 \\
\hline Escola & $4,4 \pm 2,3$ & $3,5 \pm 2,2$ & $3,0 \pm 2,2$ & 1,08 & 0,353 \\
\hline GDS & $2,4 \pm 1,5$ & $2,4 \pm 1,7$ & $2,7 \pm 1,8$ & 0,09 & 0,912 \\
\hline MEEM & $27,2 \pm 2,0$ & $25,7 \pm 1,5$ & $17,0 \pm 2,2$ & 72,27 & $0,000 *$ \\
\hline TDR & $8,9 \pm 1,6$ & $7,5 \pm 2,2$ & $3,7 \pm 1,4$ & 21,01 & $0,000 *$ \\
\hline DSD & $4,5 \pm 0,8$ & $4,4 \pm 0,7$ & $4,6 \pm 0,7$ & 0,11 & 0,898 \\
\hline DSI & $3,3 \pm 0,8$ & $3,3 \pm 0,8$ & $2,7 \pm 0,7$ & 1,40 & 0,263 \\
\hline Repai & $19,9 \pm 3,0$ & $11,3 \pm 2,2$ & $10,0 \pm 1,9$ & 47,26 & $0,000 * *$ \\
\hline Repaci & $6,3 \pm 1,4$ & $3,4 \pm 1,4$ & $0,7 \pm 1,1$ & 42,84 & $0,000 * * *$ \\
\hline
\end{tabular}

Nota. $\mathrm{CON}=$ controles; $\mathrm{CCL}=$ comprometimento cognitivo leve; DTA = demência tipo Alzheimer, $\mathrm{M}=$ média; DP = desvio-padrão, GDS = Escala de Depressão Geriátrica; MEEM = Mini Exame do Estado Mental; TDR = Teste do Desenho do Relógio; DSD = Digit Span ordem direta; DSI = Digit Span ordem indireta; Repai = recordação de palavras imediata; Repaci $=$ recordação de palavras com intervalo, $*$ CON $=$ CCL ${ }^{1}$ DTA; $* *$ CON ${ }^{1}$ CCL $=$ DTA, *** $\mathrm{CON}^{1} \mathrm{CCL}^{1}$ DTA.

A Tabela 2 apresenta as médias e os desvios-padrão dos testes de controle executivo. A ANOVA revelou diferenças estatisticamente significantes em todos os testes: RNG, Trail Making Test forma A, Trail Making Test B - A, Fluência Verbal semântica, Fluência Verbal fonológica, Reading span, Teste de Brown-Peterson e Wisconsin Card Sorting Test. Algumas exceções ocorreram em relação a algumas medidas dos testes avaliados, tais como: Trail Making Test na forma B e Teste de Brown-Peterson com dezoito segundos. A análi- se post hoc revelou que o desempenho médio nos testes de controle executivo dos idosos com DTA diferiu significativamente dos idosos controles e com CCL.

A Figura 1 apresenta os resultados do Teste de Correlação de Pearson entre os testes de memória episódica verbal (Repai e Repaci) e os testes de controle executivo. O Repai correlacionou significativamente com o Trail Making Test na forma A, com o TMT na forma B, com a Fluência Verbal semântica, com Fluência Verbal fonológica, com o Reading Span,

Tabela 2

Desempenho nos testes de Controle Executivo dos grupos controle, CCL e DTA

\begin{tabular}{|c|c|c|c|c|c|}
\hline & $\begin{array}{c}\mathrm{CON} \\
(n=13) \\
M \pm D P\end{array}$ & $\begin{array}{c}\mathrm{CCL} \\
(n=9) \\
M \pm D P\end{array}$ & $\begin{array}{c}\text { DTA } \\
(n=8) \\
M \pm D P\end{array}$ & $F$ & $p$ \\
\hline RNG & $0,42 \pm 0,10$ & $0,40 \pm 0,06$ & $0,62 \pm 0,20$ & 7,66 & $0,002 *$ \\
\hline TRIA & $108,50 \pm 53,5$ & $97,20 \pm 43,9$ & $244,90 \pm 70,1$ & 18,80 & $0,000 *$ \\
\hline TRIB & $247,50 \pm 77,5$ & $288,20 \pm 19,8$ & $300,00 \pm 0,0$ & 2,94 & 0,070 \\
\hline TRIBA & $139,00 \pm 65,7$ & $191,00 \pm 48,9$ & $55,10 \pm 70,1$ & 10,16 & $0,001 *$ \\
\hline FVfru & $11,10 \pm 2,5$ & $10,50 \quad \pm 1,8$ & $7,80 \quad \pm 3,0$ & 4,47 & $0,021 *$ \\
\hline FVfas & $27,90 \quad \pm 9,7$ & $26,10 \quad \pm 7,6$ & $16,10 \quad \pm 7,6$ & 4,93 & $0,015 *$ \\
\hline Rspan & $1,60 \pm 0,5$ & $1,20 \quad \pm 0,3$ & $1,00 \quad \pm 0,0$ & 6,70 & $0,004 *$ \\
\hline BP1 & $3,60 \pm 1,4$ & $3,60 \pm 1,1$ & $1,70 \quad \pm 1,4$ & 5,88 & $0,008 *$ \\
\hline BP3 & $3,60 \quad \pm 1,3$ & $3,30 \pm 1,0$ & $1,20 \quad \pm 1,3$ & 10,09 & $0,001 *$ \\
\hline ВР9 & $1,80 \quad \pm 1,5$ & $1,10 \quad \pm 0,9$ & $0,00 \quad \pm 0,0$ & 6,23 & $0,006 *$ \\
\hline ВР18 & $0,70 \quad \pm 1,0$ & $1,00 \quad \pm 1,4$ & $0,00 \quad \pm 0,0$ & 2,22 & 0,128 \\
\hline Wisca & $6,60 \quad \pm 1,8$ & $6,70 \quad \pm 1,7$ & $4,80 \quad \pm 0,9$ & 3,64 & 0,040 * \\
\hline Weto & $53,70 \pm 16,2$ & $51,60 \pm 15,6$ & $69,00 \pm 10,9$ & 3,50 & $0,045^{*}$ \\
\hline
\end{tabular}

Nota. $\mathrm{CON}=$ controles, $\mathrm{CCL}$ = comprometimento cognitivo leve; DTA = Demência Tipo Alzheimer; $\mathrm{M}$ = média; $\mathrm{DP}=$ desvio-padrão, RNG = Random Number Generation; TRIA = Trail Making Test forma A; TRIB = Trail Making Test forma B; TRIBA = Trail Making Test forma B - A; FVfru = Fluência verbal categoria frutas; FVfas = Fluência verbal soma das letras FAS; Rspan = Reading Span; BP1 = Teste de Brown-Peterson com um segundo; BP3 = Teste de Brown-Peterson com três segundos; BP9 = Teste de Brown-Peterson com nove segundos; BP18 = Teste de Brown-Peterson com dezoito segundos; Wisca = Wisconsin Card Sorting Test - número de categorias; Weto $=$ Wisconsin Card Sorting - erro total; ${ }^{*}$ CON $=$ CCL ${ }^{1}$ DTA . 
com o Teste de Brow-Peterson um segundo, com três segundos e com nove segundos. O Repaci correlacionou significativamente com o RNG, com o TMT forma A, com a forma B, com a Fluência Verbal semântica, com a FV fonológica, com o Reading Span, com o Teste Brown-Peterson um segundo, com três segundos e com nove segundos.

\section{Discussão}

O primeiro objetivo deste trabalho foi comparar o desempenho em alguns testes de controle executivo de idosos com Comprometimento Cognitivo Leve (CCL), idosos com Demência Tipo Alzheimer (DTA) e idosos sem déficits de memória episódica verbal. O segundo objetivo foi relacionar o comprometimento da memória episódica verbal com o desempenho nos testes de controle executivo. Os critérios e instrumentos utilizados para constituir os grupos (Entrevista Neuropsicológica, Exame do Estado Mental, GDS, MEEM, TDR e Listas de palavras - Repai e Repaci) foram adequados para discriminar indivíduos controles de indivíduos com CCL e DTA, em especial, o MEEM, o TDR, o Repai e o Repaci, tendo como referência os critérios diagnósticos do DSM-IV (Associação Psiquiátrica Americana, 1995) e da Academia Americana de Neurologia (Petersen et al., 2001).

Em nosso estudo, de cada quatro idosos avaliados, um foi excluído por apresentar sintomas depressivos. As queixas de problemas de memória em idosos estão associadas a sintomas de depressão e ansiedade e são freqüentes na população geriátrica que freqüenta ambulatórios de saúde (Green, 2000; Kizilbash, Wanderploeg, \& Curtis, 2002; Mehta, Yaffe, \& Covinsky, 2002). A análise dos resultados da amostra reve- lou que os três grupos não diferiram em relação a estes sintomas que pudessem explicar as queixas e os déficits de memória episódica verbal. Contudo, sintomas depressivos (perda de interesse, diminuição da energia e dificuldades de concentração) estão presentes em indivíduos na fase pré-clínica da DTA e estão associados à queixa subjetiva de memória (Berger, Fratiglioni, Forsell, Winbland, \& Bäckman, 1999).

O MEEM e o TDR têm sido largamente empregados em protocolos de pesquisa e em situação clínica, para rastear indivíduos com alterações cognitivas, particularmente em indivíduos com DTA e, recentemente, em indivíduos com CCL (Juby, Tench, \& Baker, 2002; Palmer et al., 2003; Petersen et al., 2001; Powlishta et al., 2002). Em nosso estudo, estes instrumentos auxiliaram na diferenciação entre os idosos avaliados. O desempenho no MEEM e no TDR diferiu significativamente entre os idosos normais e com CCL de idosos com DTA.

O comprometimento da memória episódica verbal é considerado um marcador neuropsicológico do CCL e da DTA (Collie \& Maruff, 2000; Petersen et al., 2001). A diminuição no desempenho em testes de memória episódica verbal, que ocorre no processo natural de envelhecimento, tem sido também relacionada à ativação do córtex pré-frontal (Stebbins et al., 2002; West, 1996). Em nosso estudo, o teste de recordação de uma lista de palavras adaptado da Bateria ADAS-COG (Schultz et al., 2001) diferenciou significativamente os idosos saudáveis dos idosos com CCL e DTA, evidenciando o comprometimento da memória episódica verbal nestes grupos. Alguns estudos (e.g., Bertolucci et al., 2001) têm demonstrado que medidas de aprendizado (como o Repai e o Repaci), relacionados aos processos de codificação e recuperação da memória, estão comprometidas nas fases iniciais da DTA. Estas

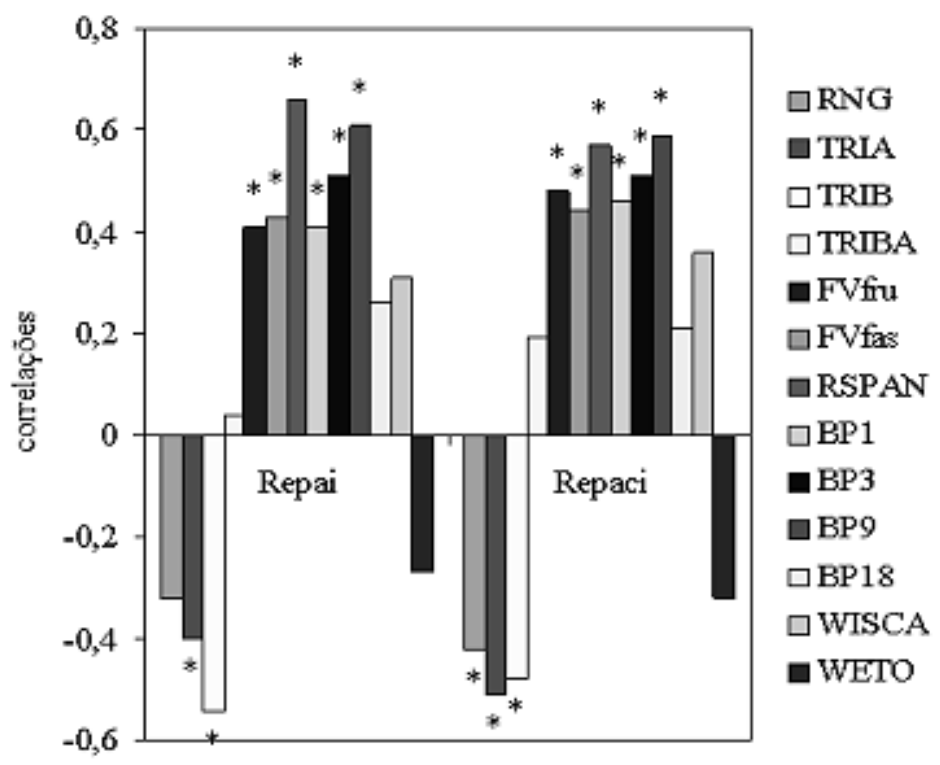

Figura 1. Valores dos coeficientes de correlação (Pearson) entre os testes de memória episódica verbal (Repai e Repaci) e os testes de controle executivo (a ordenada apresenta os valores da correlação; a abscissa indica as medidas utilizadas nos testes de controle executivo; * $p<0,05$ ). 
evidências foram corroboradas em nossa pesquisa. Tanto o desempenho no Repai quanto o desempenho no Repaci foram associados significativamente ao desempenho no MEEM e TDR, validando as medidas de recordação de palavras na discriminação de idosos com DTA e CCL.

O teste de fluência verbal tem sido largamente empregado na avaliação das FEs (Bryan \& Luszcz, 2000; Spreen \& Strauss, 1998). Em nossa investigação, o número total de palavras lembradas no teste de fluência verbal fonológica diferiu significativamente entre os grupos. Quanto à fluência verbal semântica (FAS), as diferenças também foram significantes, para os idosos com DTA. Estes achados evidenciam que nos idosos com risco maior de desenvolver demência (CCL) não há comprometimento da rede lingüística semântica e fonológica, apesar do comprometimento da memória episódica verbal.

O Trail Making Test é um dos testes neuropsicológicos mais utilizados e sensíveis à disfunção cerebral e função executiva (Spreen \& Strauss, 1998; Stuss et al., 2001). Em nosso estudo, o tempo para a resolução da tarefa na forma A foi significativamente maior para os idosos com DTA em relação aos idosos controles e com CCL. Porém, as diferenças entre os grupos CCL e controle não foram significativas, apesar do tempo maior despendido pelos idosos com CCL. Em relação ao TMT forma B, não foram observadas diferenças significantes no tempo requerido para completar a tarefa, mas todos os idosos com DTA não conseguiram completar a tarefa no tempo máximo estipulado (300 segundos). Por outro lado, quando subtraímos da forma B o tempo total da forma A, novamente a diferença foi significante somente em relação à DTA. Estes achados sugerem a presença de dificuldades maiores nos processos atencionais e na flexibilidade mental, nos estágios mais avançados do comprometimento da memória episódica verbal.

Estudos de neuroimagem sugerem que a produção de números aleatórios no teste do RNG exige considerável demanda dos processos executivos, particularmente do córtex pré-frontal dorsolateral (Jahanshahi, Dirnberg, Fuller, \& Frith, 2000). Em nosso estudo, o desempenho dos idosos com DTA no RNG foram piores, quando comparados com o desempenho dos idosos controles e com CCL, evidenciando déficits de CE naqueles idosos. Estas diferenças ocorrem provavelmente devido a dificuldades maiores dos idosos com DTA em inibir respostas estereotipadas na geração de números, processos estes que são modulados pelo córtex pré-frontal dorsolateral.

O número de categorias completadas no Wisconsin Card Sorting Test pelos idosos saudáveis foi significativamente maior em relação aos idosos com DTA, mas não em relação aos idosos com CCL. Em relação ao número total de erros cometidos, diferença significativa ocorreu, também, apenas entre os idosos controles e DTA. Estes resultados sugerem que nos estágios iniciais da presença de déficits de memória episódica (CCL) não há déficits na formação de conceitos e na flexibilidade mental. O WCST é um teste tradicionalmente empregado na avaliação da integridade dos lobos frontais (Bryan \& Luszcz, 2000; Spreen \& Strauss, 1998). Contudo, trata-se de um teste multifatorial, que requer uma rede neural distribuída, por isso, o comprometimento no desempenho deste teste pode ocorrer por vários motivos, nem todos relacionados às funções executivas (Royall et al., 2002, Spreen \& Strauss, 1998). Além disso, as medidas de avaliação utilizadas, as instruções dadas aos sujeitos e a localização da lesão podem interferir nos resultados (Stuss et al., 2000).

O Teste Reading Span tem sido relacionado com a memória operacional, em especial, com o componente executivo central (Daneman \& Carpenter, 1980, Whitney, Amett, \& Driver, 2001). Pacientes com DTA leve têm maior dificuldade no span da memória operacional e na compreensão de sentenças. Estas dificuldades estão relacionadas à diminuição da velocidade do processamento da informação, ao processamento dos recursos cognitivos do componente executivo central da memória operacional (atenção seletiva e inibição), aos aspectos semânticos (e.g., regras verbais e coerência de julgamento) e à coordenação de múltiplas tarefas cognitivas (Grossman \& Rhee, 2001; Grossman \& WhiteDevine, 1998). Em nosso estudo, esses fatores foram evidenciados no menor desempenho no teste Reading Span de pacientes com DTA em relação aos controles.

O Teste Brown-Peterson é utilizado para avaliar a memória operacional e a capacidade de processamento de informação por meio da retenção de poucos itens num curto período de tempo (Folden, Stuss, \& Craik, 2000; Spreen \& Strauss, 1998). Estudos recentes de neuroimagem têm evidenciado que regiões do córtex pré-frontal são responsáveis pela manipulação e manutenção da informação por um curto período de tempo, e que estas regiões estão envolvidas nos processos de codificação e recuperação da memória episódica (Daselaar, Veltman, Rombouts, Raaijmakers, \& Jonker, 2003; Nyberg et al., 2003; Ranganath, Johnson, \& D’Esposito, 2003). Portanto, déficits de memória episódica verbal, avaliados por meio de medidas de retenção e recuperação, provavelmente estão associados a déficits de controle executivo. Em nosso estudo, diferenças significativas ocorreram nas medidas de tempo iniciais (1, 3 e 9 segundos) em relação à DTA, evidenciando comprometimento no armazenamento da informação de curto prazo.

Em síntese, a análise das relações entre memória episódica verbal e testes de controle executivo em idosos com DTA e CCL revelou que, apesar de ambos apresentarem comprometimento em tarefas de recordação de palavras, tanto para codificação quanto para recuperação, idosos com CCL não apresentam déficits em tarefas que avaliam o controle executivo, apesar das tarefas de controle executivo correlacionarem significativamente com as tarefas de memória episódica verbal. O conjunto destes resultados possibilitou compreender melhor a relação entre déficits de memória episódica verbal e controle executivo. Contudo, outros estudos são necessários, em especial, sobre o armazenamento e a manipulação temporária da informação, para que possamos compreender melhor a relação entre o declínio da memória episódica verbal e o controle executivo, no processo de transição entre o envelhecimento cognitivo normal e o patológico. 


\section{Referências}

American Psychological Association. (1998). Presidential Task Force on the assessment of Age-consistent Memory Decline and Dementia: guidelines for the evaluation of dementia and age-related cognitive decline. American Psychogicologist, 53(12), 1298-1303.

Associação Psiquiátrica Americana. (1995). Manual diagnóstico e estatístico de transtornos mentais ( $4^{\underline{\underline{a}}}$ ed.). Porto Alegre: Artes Médicas.

Barnes, L. L., Wilson, R. S., Schneider, J. A., Bienias, J. L., Evans, D. A., \& Bemmett, D. A. (2003). Gender, cognitive decline, and risk of AD in older persons. Neurology, 60, 1777-1781.

Berger, A-K., Fratiglioni, L., Forsell, Y., Winbland, B., \& Backman, L. (1999). The occurrence of depressive symptoms in the preclinical phase of AD: a population-based study. Neurology, 53(9), 1998-2002.

Bertolucci, P. H. F., Okamoto, I. V., Brucki, S. M. D., Siviero, M. O. Toniolo Neto, J., \& Ramos, L. R. (2001). Applicability of the CERAD neuropsychological battery to Brazilian elderly. Arquivos de Neuropsiquiatria, 59(3A), 532-536.

Binetti, G., Magni, E., Padovani, A., Cappa, S. F., Bianchetti, A., \& Trabuccho, M. (1996). Executive dysfunction in early Alzheimer's Disease. Journal of Neurology, Neurosurgery and Psychiatry, 60, 91-93.

Bischkop, J., Busse, A., \& Angermeyer, M. C. (2002). Mild cognitive impairment - a review of prevalence, incidence and outcome according to current approaches. Acta Psychiatrica Scandinavica, 106, 403-414.

Bryan, J., \& Luszcz, M. A. (2000). Measurement of executive function: considerations for detecting adult age differences. Journal of Clinical and Experimental Neuropsychology, 22(1), 40-55.

Brown, J. (1958). Some tests of decay of immediate memory. Quarterly Journal of Experimental Psychology, 10, 12-21.

Buerger, K., Teipel, S. J., Zinkowski, R., Blennow, K., Arai, H., Engel, R., Hofman-Kiefer, K., McCulloch, C., Ptok, U., Heun, R., Andrreasen, N., DeBernardis, J., Kerkman, D., Moeller, H.-J., Davies, P., \& Hampel, H.. (2002). CSF tau protein phosphorylated at theonine 231 correlates with cognitive decline in MCI subjects. Neurology, 59, 627-629.

Carpenter, P. A., Just, M. A., \& Reichle, E. D. (2000). Working memory and executive function: evidence from neuroimaging. Current Opinion of Neurobiology, 10(2), 195-199.

Collie, A., \& Maruff, P. (2000). The neuropsychology of preclinical Alzheimer's disease and mild cognitive impairment. Neuroscience and Biobehavioral Reviews, 24, 365-374.

Daneman, M., \& Carpenter, P. A. (1980). Individual differences in working memory and reading. Journal of Verbal Learning and Verbal Behavior, 19, 450-466.

Daselaar, S. M., Veltman, D. J., Rombouts, S. A. R. B., Raaijmakers, J. G. W., \& Jonker, C. (2003). Neuroanatomical correlates of episodic encoding and retrieval in young and elderly subjects. Brain, 126, 43-56.

Faw, B. (2003). Pre-frontal executive committee for perception, working memory, attention, long-term memory, motor control, and thinking: a tutorial review. Consciouness and Cognition, 12, 83-139.

Folden, D., Stuss, D. T., \& Craik, F. I. M. (2000). Age differences in performance on two versions of the Brown-Peterson Task. Aging, Neuropsychology, and Cognition, 7(4), 245-259.

Folstein, M. F., Folstein, S. E., \& Mchugh, P. R. (1975). Mini-mental state: a practical method for grading the cognitive state of patients for the clinician. Journal of Psychiatric Research, 12(3), 189-198.

Foster, J. K., Behrmann, M., \& Stuss, D. T. (1999). Visual attention deficits in Alzheimer's disease: simple versus conjoined feature search. Neuropsychology, 13(2), 223-245.

Funahashi, S. (2001). Neuronal mechanisms of executive control by the prefrontal cortex. Neuroscience Research, 39, 147-165.

Grady, C. L., \& Craik, F. I. M. (2000). Changes in memory with age. Current Opinion in Neurobiology, 10, 224-231.
Green, J. (2000). Neuropsychological evaluation of the older adult: a clinician's guidebook. San Diego: Academic.

Grossman, M., \& Rhee, J. (2001). Cognitive resources during sentence processing in Alzheimer's disease. Neuropsychologia, 39, 1419-1431.

Grossman, M., \& White-Devine, T. (1998). Sentence comprehension in Alzheimer's disease. Brain and Language, 62, 186-201.

Hamdan, A. C., Bueno, O. F. A., \& Souza, J. A. (2004). Performance of university students on random number generation at different rates to evaluate executive functions. Arquivos de Neuropsiquiatria, 62(1), 58-60.

Hänninen, T., Hallikainen, M., Koivisto, K., Partanen, K., Laakso, M. P., Riekkinen, P.J., \& Soininen, H. (1997). Decline of frontal lobe functions in subjects with age-associated memory impairment. Neurology, 28, 148-153.

Jahanshahi, M., Dirnberg, G., Fuller, R., \& Frith, C. D. (2000). The role of the dorsolateral prefrontal cortex in random number generation: a study with positron emission tomography. Neuroimage, 12(6), 713-724.

Juby, A., Tench, S., \& Baker, V. (2002). The value of clock drawing in identifying executive cognitive dysfunction in people with a normal MiniMental State Examination score. Canadian Medical Association Journal, 167(8), 859-864.

Kizilbash, A. H., Vanderploeg, R. D., \& Curtiss, G. (2002). The effects of depression and anxiety on memory performance. Archives of Clinical Neuropsychology, 17, 57-67.

Mehta, K. M., Yaffe, K., \& Covinsky, K. (2002). Cognitive impairment, depressive symptoms, and functional decline in older people. Journal of American Geriatric Society, 50, 1045-1050.

Morris, J. C. (1993). The Clinical Dementia Rating (CDR): current version and scoring rules. Neurology, 43, 2412-2414.

Morris, R. G. (1994). Working memory in Alzheimer-type dementia. Neuropsychology, 8(4), 544-554.

Nyberg, L., Marklund, P., Persson, J., Cabeza, R., Forkstam, C., Petersson, M., \& Ingvar, M. (2003). Common prefrontal activations during working memory, episodic memory, and semantic memory. Neuropsychologia, 41, 371-377.

Palmer, K., Bäckman, L., Winblad, B., \& Fratiglioni, L. (2003). Detection of Alzheimer's disease and dementia in the preclinical phase: population based cohort study. British Medical Journal, 326, 245-250.

Perry, R. J., \& Hodges, J. R. (1999). Attention and executive deficits in Alzheimer's disease. A critical review. Brain, 122, 383-404.

Petersen, R. C., Smith, G. E., Waring, S. C., Ivnik, R. J., Tangalos, E., \& Kolmen, E. (1999). Mild Cognitive Impairment: clinical characterization and outcome. Archives of Neurology, 56, 303-308.

Petersen, R. C., Steves, J. C., Ganguli, M., Tangalos, E. G., Cummings, J. L., \& DeKosky, S. T. (2001). Practice parameter: early detection of dementia; mild cognitive impairment (an evidence-based review). Neurology, 56, 1133-1142.

Peterson, L. R., \& Peterson, M. J. (1959). Short-term retention of individual verbal items. Journal of Experimental Psychology, 58, 193-198.

Powlishta, K. K., Von Dras, D. D., Stanford, A., Carr, D. B., Tsering, C., Miller, J. P., \& Morris, J. C. (2002). The clock drawing test is a poor screen for very mild dementia. Neurology, 59, 898-903.

Ranganath, C., Johnson, M. K., \& D’Esposito, M. (2003). Prefrontal activity associated with working memory and episodic long-term memory. Neuropsychologia, 41, 378-389.

Riemenschneider, M., Lautenschalager, N., Wagenpfeil, S., Diehl, J., Drzezga, A., \& Kurz, A. (2002). Cerebrospinal fluid Tau and ß-Amyloid 42 proteins identify Alzheimer disease in subjects with Mild Cognitive Impairment. Archives of Neurology, 59, 1729-1734.

Royall, D. R., Lauterbach, E. C., Cummings, J. L., Reeve, A., Rummans, T. A., Kaufer, D. I., LaFrance, W. C., \& Coffrey, C. E. (2002). Executive control function: a review of its promise and challenges for clinical research. A report from the Committee on Research of the American Neuropsychiatric Associations. Journal of Neuropsychiatry Clinical Neuroscience, 14(4), 377-405. 
Schultz, R. R., Siviero, M. O., \& Bertolucci, P. H. F. (2001). The cognitive subscale of the "Alzheimer's Disease Assessment Scale” in a Brazilian sample. Brazilian Journal of Medical and Biological Research, 34, 1295-1302.

Smith, E. E., \& Jonides, J. (1999). Storage and executive process in the frontal lobes. Science, 283(5408), 1657-1661.

Spreen, O., \& Strauss, E. (1998). A compendium of neuropsychological tests. Administration, norms, and commentary. Nova York: Oxford University Press.

Stebbins, G. T., Carrillo, M. C., Dorfman, J., Dirkse, C., Desmond, J. E., Turner, D. A., Bennett, D. A., Wilson, R. S., Glover, G., \& Gabrielli, D. E. (2002). Aging effects on memory encoding in the frontal lobes. Psychology and Aging, 17(1), 44-55.

Stuss, D. T., \& Alexander, M. P. (2000). Executive functions and the frontal lobes: a conceptual view. Psychological Research, 63, 289-298.

Stuss, D. T., Bisschop, S. M., Alexander, M. P., Levine, B., Katz, D., \& Izukawa, D. (2001). The Trail Making Test: a study in focal lesions patients. Psychological Assessment, 13(2), 230-239.

Stuss, D. T., \& Levine, B. (2002). Adult clinical neuropsychology: lessons from studies of the frontal lobes. Annual Review of Psychology, 53, 401-433.

Stuss, D. T., Levine, B., Alexander, M. P., Hong, J., Palumbo, C., Hamer, L., Murphy, K. L., \& Izukawa, D. (2000). Wisconsin Card Sorting Test performance in patients with local frontal and posterior brain damage: effects of lesion and test structure on separable cognitive process. Neuropsychologia, 38, 388-402.
Tekin, S., \& Cummings, J. L. (2002). Frontal-subcortical neuronal circuits and clinical neuropsychiatry: an update. Journal of Psychosomatic Research, 53, 647-654.

Tirapu-Ustárroz, J., Muñoz-Céspedes, J. M., \& Pelegrín-Valero, C. (2002). Funciones ejecutivas: necessidad de uma integración conceptual. Revista de Neurología, 34, 673-685.

Trzepacz, P. T., \& Baker, R. W. (1993). The psychiatric mental status. Nova York: Oxford University Press.

Wechsler, D. (1981). Wechsler Adult Intelligence Scale - revised. Nova York: Psychological Corporation.

West, R. L. (1996). An application of prefrontal cortex function theory to cognitive aging. Psychological Bulletin, 120(2), 272-292.

Whitney, P., Arnett, P. A., \& Driver, A. (2001). Measuring central executive functioning: what's in a reading span. Brain and Cognition, 45(1), 1-14.

Woodruff-Pak, D. (1997). The neuropsychology of aging. Oxford: Blackwell.

Yesavage, J. A., Brink, T. L., Rose, T. L., Lum, O., Huang, V., Adey, M. B., \& Leirer, V. O. (1983). Development and validation of a geriatric depression screening scale: a preliminary report. Journal of Psychiatric Research, 17, 37-49.

Yuspeh, R. L., Vanderploeg, R. D., Crowell, T. A., \& Mullan, M. (2002). Differences in executive functioning between Alzheimer's disease and subcortical ischemic vascular dementia. Journal of Clinical and Experimental Neuropsychology, 24(6), 745-754.

Amer Cavalheiro Hamdan, Doutor em Psicobiologia pela Universidade Federal de São Paulo, é professor adjunto no Departamento de Psicologia da Universidade Federal do Paraná. Endereço para correspondencia: Rua Atílio Borio, 174, apto 114; Bairro Cristo Rei; Curitiba, PR; CEP 80050-250. Tel.: (41) 3262-8760. Email: achamdan@ufpr.br

Orlando Francisco Amodeo Bueno, livre-docente pela Universidade Federal de São Paulo, é professor titular no Departamento de Psicobiologia da Universidade Federal de São Paulo-UNIFESP. 